\title{
Attending to a misoriented word causes the eyeball to rotate in the head
}

\author{
HAROLD PASHLER and V. S. RAMACHANDRAN \\ University of California, San Diego, California \\ and \\ MARK W. BECKER \\ Lewis and Clark College, Portland, Oregon
}

\begin{abstract}
Torsional eye movements are triggered by head tilt and a rotating visual field. We examined whether attention to a misoriented form could also induce torsion. Thirty-six observers viewed an adapting field containing a bright vertical line, and then they viewed a display that was composed of two misoriented words (one rotated clockwise, the other counterclockwise, by $15^{\circ}, 30^{\circ}$, or $45^{\circ}$ ). The subjects were instructed to attend to one of the words. The subjects' adjustments of a reference line to match the tilt of the afterimage showed that attention to a misoriented word produces torsional eye movement (verified with direct measurements on 4 additional subjects). This eye movement reduces the retinal misorientation of the word by about $1^{\circ}$. The results of this study reinforce the linkage between selective attention and eye movements and may provide a useful tool for dissecting different forms of "mental rotation" and other adjustments in internal reference frames. Apparentmotion displays confirming that the eye rotated in the head may be downloaded from www.psychonomic.org/archive.
\end{abstract}

When the human head is rotated about the line of sight, the eye rotates slightly in its socket, a response sometimes referred to as "countertorsion" (Howard, 1986). In animals such as rabbits, the response is often large enough to keep the retinal meridian upright. In human beings, however, the response is rarely greater than $6^{\circ}-8^{\circ}$ (Miller, 1962). Previous research has shown that when viewing a large stimulus rotating about the line of sight, people also experience a torsional ocular response in the direction of the rotation (Brecher, 1934; Dichgans, Held, Young, \& Brandt, 1972). This visually induced countertorsion is small $\left(1^{\circ}-4^{\circ}\right)$, and its magnitude depends on the size of the rotating stimulus (Wade, Swanston, Howard, Ono, \& Shen, 1991). Its effect on the eyes appears to combine with the effects of head tilt in an approximately additive fashion (Merker \& Held, 1981). Torsional movements have also been elicited by a sort of biofeedback training (Balliet \& Nakayama, 1978).

In the present study, we asked whether merely attending to a static word that is misoriented relative to its fa-

This work was supported by NSF Grant SBR 9729778 and NIMH Grant R01-MH45584. The authors thank Noriko Coburn, Chris Liew, and Trung Nguyen for their technical assistance, and Ian Chapman and Gurkirit Jhawar for their help with data collection. We also thank Jim Hoffman, who provided helpful comments on an earlier draft of the manuscript. Address correspondence to H. Pashler, Department of Psychology, Univarsity of California, San Diego, 9500 Gilman Drive 0109, La Jolla, CA 92093 (e-mail: hpashler@ucsd.edu). miliar upright orientation might also produce torsional eye movements that tend to correct for the misorientation. People take longer to identify misoriented familiar stimuli than they do upright stimuli, even with simple forms like letters and digits (Jolicœur \& Landau, 1984; Jolicœur, Snow, \& Murray, 1987). This length of time is substantially greater for more complex stimuli, such as words (Koriat \& Norman, 1984) and faces (Johnston, Hill, \& Carman, 1992). Misorientation in retinal instead of gravitational coordinates appears critical in producing the slowed recognition (Corballis, 1988).

To examine the effects of reading a stationary misoriented word, we had observers - with their heads stabilized - view an adapting field designed to generate an afterimage at the vertical midline. Next they viewed a display composed of two superimposed words, both centered at fixation-one rotated $15^{\circ}, 30^{\circ}$, or $45^{\circ}$ clockwise, and the other rotated to the same extent counterclockwise. A verbal instruction indicated which word should be attended to, and the subject adjusted a vertical reference line to match the orientation of the afterimage. The use of the two-word display served two purposes. First, because the display was bilaterally symmetrical, no torsional eye movements could be attributed to the retinal stimulus. Second, by instructing the subject to attend to one stimulus only, we were able to ask whether selective attention gates information flow in the pathways subserving ocular torsion. 


\section{METHOD}

\section{Subjects}

The subjects were 33 undergraduates from the University of California, San Diego.

\begin{abstract}
Apparatus and Stimuli
The stimuli were presented on 17-in. Sony Trinitron Multiscan 17SEII CRT monitors controlled by IBM personal computers. The adapting field consisted of a black field with a white vertical midline $0.4 \mathrm{~cm}$ wide $\left(.4^{\circ}\right.$ visual angle, based on a viewing distance of $60 \mathrm{~cm}$ ) running the entire vertical extent of the display. The fixation point was a red dot $0.5 \mathrm{~cm}$ in diameter. The test displays were composed of two words plus a $0.1-\mathrm{cm}$ reference line presented on a light-gray background (Figure 1). The words were all five letters long and ranged in length from 3.5 to $4.3 \mathrm{~cm}$. Test displays consisted of two words symmetrically disoriented (one clockwise, the other counterclockwise) by $15^{\circ}, 30^{\circ}$, or $45^{\circ}$. There were six words: three green (HELLO, RABBIT, and HUMAN) and three red (WORLD, OFFICE, and FOREST).
\end{abstract}

\section{Procedure}

The subjects rested their heads on a chin rest with a head stabilizer (Richmond Products, Model HCRD2R). They were told to keep their heads absolutely still, and the experimenter ascertained that no visible head movements took place. On each trial, the observer viewed the adapting field for $20 \mathrm{sec}$. Next, he or she saw the test field. As the display appeared, the computer played a sound file naming one of the two words in the display and the subject was instructed to attend to this word. While doing so, the subject adjusted the orientation of the reference line by pressing the "N" or " $M$ " key to rotate the line $1^{\circ}$ counterclockwise or clockwise, respectively. The subjects were told to adjust the tilt of the reference line to match the tilt of the "ghostly line" (or its maximum tilt, in the event it fluctuated). The subjects then viewed the test field for $8 \mathrm{sec}$, hitting the space bar if they were satisfied that they had matched the tilt of the afterimage.

\section{RESULTS AND DISCUSSION}

Data from one subject who reported seeing no afterimage at all were discarded. We conducted a $2 \times 3$ repeated measures ANOVA with 2 levels of direction of stimulus rotation (counterclockwise and clockwise) and 3 levels of degree of rotation $\left(15^{\circ}, 30^{\circ}\right.$, and $\left.45^{\circ}\right)$ as independent variables. The angular orientation of the reference line was the dependent variable. The analysis revealed a main effect of direction of stimulus rotation $[F(1,36)=5.9, p=$ $.02]$. When subjects attended to a word that was rotated in a given direction, the result was an eye position that was rotated in the same direction. Neither the main effect of degree of stimulus rotation nor the interaction between degree of rotation and direction of rotation reached significance (both $F \mathrm{~s}<1$ ). Figure 2 shows the mean eye position that was elicited when subjects switched attention from one stimulus to another at different orientations. The attention shift from left- to right-oriented words elicited torsional eye movements about $2^{\circ}$ in magnitude. Many subjects reported that the magnitude of this rotation exhibited slow fluctuations despite the subjects' continued attention to the misoriented word. The visually driven countertorsion that is induced by rotating the visual field has also been found to fluctuate, whereas the effect produced by head tilt seems much less variable (Merker \& Held, 1981).

To be certain that the attention shifts were inducing torsional eye movements instead of tiny head movements that might be going unnoticed by either the subjects or the experimenter, we performed a more direct verification with

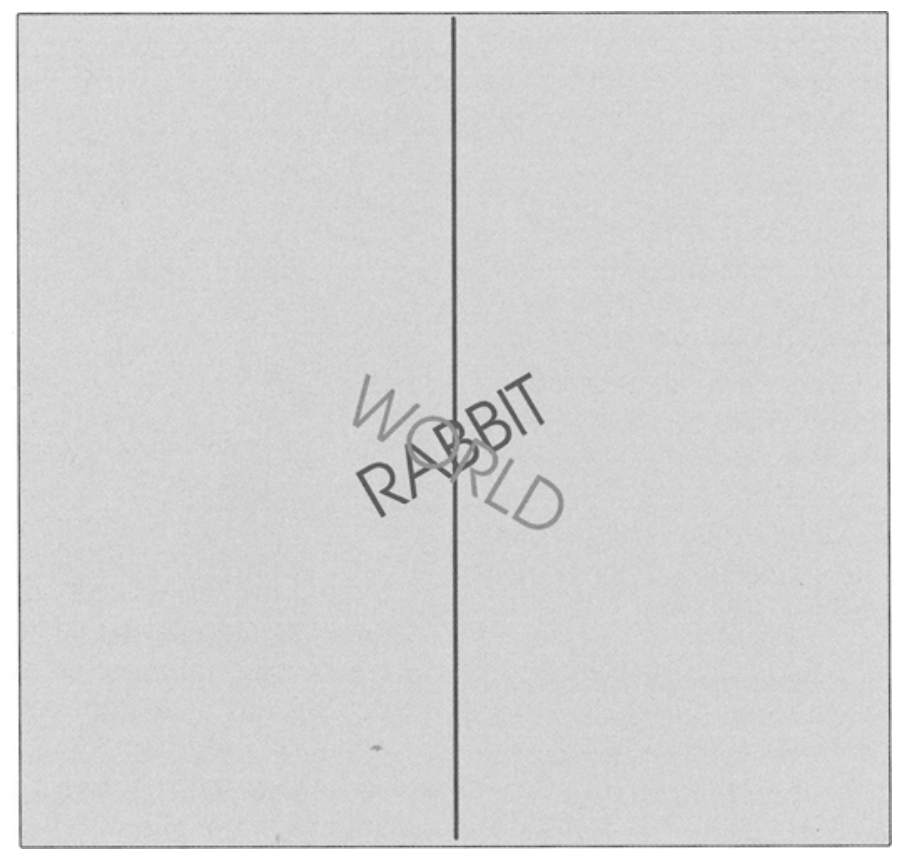

Figure 1. Sample test display, consisting of two words (one red, wORLD; the other green, RABBIT) and a vertical reference line. The observer used the keyboard to adjust the rotation of the reference line to match the afterimage created by the preceding adapting display. 


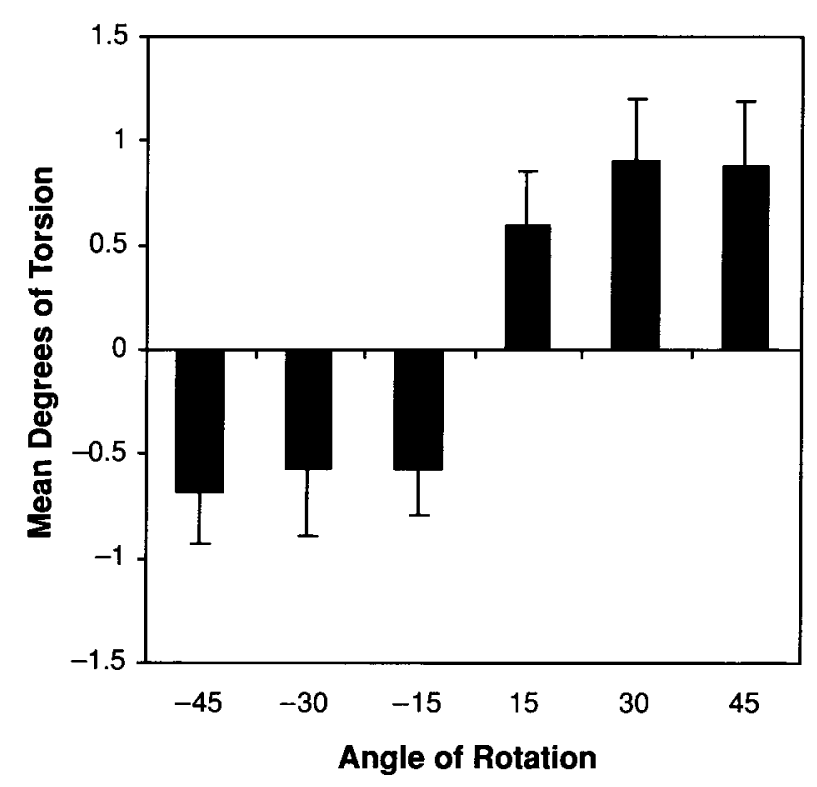

Figure 2. Mean extent of torsional eye movement as a function of the orientation of the attended stimulus.

4 different observers. Four ink marks were drawn upon the face of each of the new observers to provide visual landmarks of head position. The observers then viewed a display composed of a series of frames, each containing two single-word names of U.S. states. One of the state names was red and the other was green; one was oriented at $+45^{\circ}$ and the other at $-45^{\circ}$. Each frame was presented for $1 \mathrm{sec}$ and immediately replaced with two new state names. Subjects were instructed to attend to either the red or the green word throughout the entire procedure. Between each pair of words there was a $1 / 3$ probability that the color would switch from one orientation to the other. On these trials, the instructions obliged the subject to switch his or her attention from $-45^{\circ}$ to $+45^{\circ}$ or vice versa.

The upper portion of the observers' faces (including the eyes and the dots on the face) were videotaped while the observers viewed these displays. To verify the occurrence of torsional eye movements, single frames of video were captured before $(\sim 500 \mathrm{msec})$ and after $(\sim 500 \mathrm{msec})$ a change in orientation. The landmarks on the face were aligned in these two images, and the two images were placed into apparent-motion displays (i.e., two-frame animations). These animations, which appear to eliminate any doubts about whether the eyes actually rotate relative to the head, can be viewed online. The reader can access these at www.psychonomic.org/archive/.

Informal observations suggested some further points to consider. When observers viewed a single word without receiving instructions to attend to or even read the word, torsional eye movements often failed to occur (an observation which may possibly challenge the claim that word reading is completely automatic; see, e.g., LaBerge, 1980). Similarly, when observers viewed a square without receiving specific instructions, torsional eye movements were rarely noted. However, when observers viewed a square and attempted to see it as a diamond with the top to the right or left, eye rotation was regularly triggered. Thus, it seems that the subjects' intent to assign a top-bottom axis to the form was critical to the production of eye rotation. It was also noted that torsional eye movements seemed to continue during protracted viewing of stimuli that were as simple as a single letter. This is rather interesting because, for letters-unlike words-misorientation produces only a very tiny effect on identification time (Joliccur, Snow, \& Murray, 1987). Thus, one could infer that cyclotorsion is not a one-shot process that occurs only until stimulus identification has been completed; rather, it seems to be associated with the ongoing maintenance of an off-vertical subjective frame of reference.

One very intriguing question about torsional eye movements arises: What function, if any, might these movements serve in man? After all, torsional eye movements generally eliminate no more than a small percentage of the actual rotation induced by stimulus misorientation or head tilt. It is hard to see how such a change would provide more than a negligible benefit for visual performance. The present results, which suggest that top-down influences can also drive torsional eye movements, make the question of function even more mysterious. If the torsional response to head tilt and image rotation are vestigial holdovers from visual systems with greater rotational mobility, can the same be said of the top-down influences examined here?

However puzzling these influences may be from a functional standpoint, the results are well in line with the emerging consensus that visual selective attention is tightly tied to the control of eye movements - a point that has been well documented for saccadic, smooth pursuit, and vergence eye movements (see Hoffman, 1998, and Pashler, 1998, for a review). The phenomenon of cognitively driven torsional eye movements may also offer a useful method for analyzing the perceptual and cognitive operations used to process misoriented stimuli. The term "mental rotation" has been used (e.g., by Rock, 1973) to encompass the meaning of what happens when an observer identifies a familiar object despite its misorientation (as in the present task) and what happens when an observer judges whether a rotated letter is a mirror image or normal letter, as in the classic mental rotation task (Cooper $\&$ Shepard, 1984). It is certainly tempting to imagine that these processes are the same. However, Corballis (1988) has argued that the two kinds of tasks require quite different mental operations. Identifying a misoriented stimulus may involve assigning a top-bottom axis to the object, without imagining any transformation of its position with respect to the viewer's body. On the other hand, making a normal versus mirror image judgment may require imagining the object rotated upright with respect to the viewer's body. If torsional eye movements are triggered by a mismatch between the retinal top-bottom axis and the perceptually assigned top-bottom axis, then examining the time course of these eye movements in various mental rotation tasks may reveal important dissociations. 
Following Corballis's analysis, one could conjecture that the tasks that involve "mental rotation" might turn out not to elicit any physical rotation of the eyeballs in the head, whereas tasks that involve seeing an object with respect to a frame of reference distinct from the retinal vertical may elicit such movements. Thus, the phenomenon reported here could possibly turn out to be a useful tool, as well as a puzzling curiosity.

\section{REFERENCES}

Balliet, R., \& NaKayama, K. (1978). Egocentric orientation is influenced by trained voluntary cyclorotary eye movements. Nature, 275, 214-216.

Beintema, J. A., \& van den Berg, A. V. (2000). Perceived heading during simulated torsional eye movements. Vision Research, 40, 549-566.

BRECHER, G. A. (1934). Die optokinetische Auslösung von Augenrollung und rotatorischem Nystagmus. Pflügers Archiv für die gesamte Physiologie des Menschen und der Tiere, 234, 13-28.

COOPER, L. A., \& SHEPARD, R. N. (1984). Turning something over in the mind. Scientific American, 251, 106-114.

Corballis, M. C. (1988). Recognition of disoriented shapes. Psychological Review, 95, $115-123$.

Dichgans, J., Held, R., Young, L. R., \& BrandT, T. (1972). Moving visual scenes influence the apparent direction of gravity. Science, 178, 1217-1219.

HoFfman, J. E. (1998). Visual attention and eye movements. In H. Pashler (Ed.), Attention (pp. 119-153). Hove, U.K.: Psychology Press.

HowARD, I. P. (1986). The perception of posture, self motion, and the visual vertical. In K. R. Boff, L. Kaufman, and J. P. Thomas (Eds.), Handbook of perception and human performance (Vol. I, pp. 1-62). New York: Wiley-Interscience.

Hughes, P. C., Brecher, G. A., \& Fishkin, S. M. (1972). Effects of rotating backgrounds upon the perception of verticality. Perception \& Psychophysics, 11, 135-138.

Johnston, A., Hill, H., \& Carman, N. (1992). Recognizing faces: Effects of lighting direction, inversion, and brightness reversal. Perception, 21, 365-375.

Jolicaur, P., \& LANDAU, M. J. (1984). Effects of orientation on the identification of simple visual patterns. Canadian Journal of Psychology, 38, 80-93.

JolickUR, P., SNOW, D., \& MuRRaY, J. (1987). The time to identify disoriented letters: Effects of practice and font. Canadian Journal of Psychology, 41, 303-316.

KORIAT, A., \& NORMAN, J. (1984). What is rotated in mental rotation? Journal of Experimental Psychology: Learning, Memory, \& Cognition, 10, $421-434$.

LABERGE, D. (1980). Unitization and automaticity in perception. Nebraska Symposium on Motivation, 28, 53-71.

MERKER, B. H., \& HELD, R. (1981). Eye torsion and the apparent horizon under head tilt and visual field rotation. Vision Research, 21, 543-547.

MiLLER, E. F., II (1962). Counterrolling of the human eyes produced by head tilt with respect to gravity. Acta Otolaryngologica, 54, 479-501.

PASHLER, H. E. (1998). The psychology of attention. Cambridge, MA: MIT Press.

Rock, I. (1973). Orientation and form. New York: Academic Press.

Wade, N. J., Swanston, M. T., Howard, I. P., Ono, H., \& Shen, X. (1991). Induced rotary motion and ocular torsion. Vision Research, 31, 1979-1983.

\section{ARCHIVED MATERIALS}

The following materials associated with this article may be accessed through the Psychonomic Society's Norms, Stimuli, and Data archive, www.psychonomic.org/archive.

To access these files, search the archive for this article using the journal name (Psychonomic Bulletin \& Review), the first author's name (Pashler), and the publication year (2006).

FILE: Pashler-PB\&R-2006.zip

DESCRIPTION: The compressed archive file contains 4 files:

PashlerEtAl(2006).gif, containing demo 1 clockwise rotation PashlerEtAl(2006).gif, containing demo 2 clockwise rotation PashlerEtAl(2006).gif, containing demo 3 clockwise rotation PashlerEtAl(2006).gif, containing demo 4 clockwise rotation AUTHOR's E-MAIL ADDRESs: hpashler@ucsd.edu.

(Manuscript received July 18, 2005; revision accepted for publication April 8, 2006.) 